\title{
Reactive-Based Position Control of an Underactuated Quadrotor
}

\author{
Tom Stian Andersen \\ Department of Electrical Engineering \\ The Arctic University of Norway \\ NO-8514 Narvik, Norway \\ Email: tom.s.andersen@uit.no
}

\author{
Raymond Kristiansen \\ Department of Electrical Engineering \\ The Arctic University of Norway \\ NO-8514 Narvik, Norway \\ Email: raymond.kristiansen@uit.no
}

\begin{abstract}
This paper addresses the problem of position control or waypoint tracking for an underactuated quadrotor. The proposed control law is what is known as reaction based in the way that the attitude system reacts to errors in the translational motion. This methodology requires no generation of desired attitude or angular velocity and the resulting control law is model-independent in that it does not require the knowledge of the inertia matrix. In addition the controller has a very simple structure making it suitable for small quadrotor platforms. Simulation results are provided and discussed to demonstrate the proposed method.
\end{abstract}

\section{INTRODUCTION}

In recent years, Quadrotors have received a lot of attention from researchers. Partly because of their simple mechanical structure, low cost and high maneuverability, which makes them suitable for a wide range of applications [1], [2]. However, they come with their own challenges such as instability in the absence of feedback, highly nonlinear dynamics, tight coupling between rotational and translational motion and underactuation. Often feedback control is done using linear techniques such as PID control [3], [4], [5] because they are simple to implement and they yield good performance [6]. In applications that require high-performance where quadrotors operate near their physical limits it becomes necessary to use nonlinear control strategies [1].

In several previous works on nonlinear quadrotor control the focus is on the trajectory tracking problem where a virtual position controller is first constructed assuming that the quadrotor is fully actuated in its translational motion. Then a desired orientation is extracted from the force vector generated by the virtual position controller which is then tracked by an attitude controller [7]. These approaches have to deal with the topological obstructions of the orientation manifold [8], which can lead to more complicated control structures. Another approach that circumvents the topological obstructions of the orientation manifold are dynamics extension techniques that rely on taking the derivative of the thrust until three independent control inputs appear in the translational equations of motion [9]. However, these techniques present challenges in that it becomes necessary to take motor dynamics into consideration. In [10] the thrust vector is only differentiated once and then the angular velocities are seen as a virtual control input that is tracked by an attitude controller. The derived attitude controller however, requires the derivative of the desired angular velocity which can be difficult to implement. In [11] a second order sliding mode attitude controller is derived that exponentially stabilizes the attitude at hover. A PD-controller for the translational error is derived and is used to inject angular accelerations into the rotational dynamics to perturb the system towards the desired trajectory. In both works when the quadrotor is far away from its desired position very large angular accelerations will be injected into the rotational dynamics which can lead to a highly oscillatory response. In addition, when the position errors become small the robust controller will start to reject the injected angular accelerations. In [12] a model-free robust attitude controller built on differintegral fractional operators is developed, which reacts to injected disturbances from the positional system.

In this paper a reactive controller is developed that in concept is similar to [11] and [12]. There are however, some key differences in that there is no desired orientation for the attitude dynamics and the injected translational control signals are saturated. The control design works in two steps, first a backstepping position controller is derived that generates a desired force that when tracked makes the equilibrium of the error dynamics uniformly globally asymptotically stable. Secondly, the desired force is then saturated and injected into the attitude dynamics as a torque together with a dampening term on the angular velocities. The dampening term ensures boundedness of the angular velocities while the injected torque consequently tilts the quadrotor's thrust axis in the direction of the desired force. This results in a very simple control structure which is suitable for implementation on small quadrotors with limited processing power. The attitude controller is free of ambiguities and singularities, which usually occurs when designing attitude controllers on $\mathrm{SO}(3)$. Additionally the attitude controller is model-independent in that it doesn't require the knowledge of the inertia matrix.

This paper is organized in the following way. Section II introduces notation and some useful properties of quaternions. Section III details the dynamic model for the quadrotor that uses quaternions for the attitude parameterization. Section IV derives the position controller and the reactive-based attitude 
controller which is the main result of the paper. In Section $\mathrm{V}$ simulations are performed to show the potential of the approach and a brief conclusion is given in Section VI highlighting some possible extensions of this work.

\section{Preliminaries}

\section{A. Notation and reference frames}

Vectors are denoted as lower-case bold letters while scalars are non-bold for instance $\boldsymbol{x} \in \mathbb{R}^{n}$ is an n-dimensional vector while $a \in \mathbb{R}$ is a scalar. Matrices are upper-case bold letters where the transpose of an $n \times m$ matrix $\boldsymbol{M} \in \mathbb{R}^{n \times m}$ is denoted by $\boldsymbol{M}^{\top}$. The $n \times n$ identity matrix is denoted as $\boldsymbol{I}_{n \times n}$ while an $n \times m$ matrix with zero entries is denoted as $\mathbf{0}_{n \times m}$. The unit vector $\boldsymbol{e}_{3}$ is defined as $\boldsymbol{e}_{3}=\left[\begin{array}{lll}0 & 0 & 1\end{array}\right]^{\top}$. The derivative with respect to time is denoted as $\dot{\boldsymbol{x}}=\frac{d \boldsymbol{x}}{d t}$. Reference frames are denoted as $\mathcal{F}_{(\cdot)}$, and superscripts are used to denote a vector frame of reference, such that the vector $\boldsymbol{x}^{A}$ is expressed in $\mathcal{F}_{A}$. Angular velocities are denoted as $\boldsymbol{\omega}_{A, B}^{C} \in \mathbb{R}^{3}$ which is the angular velocity of $\mathcal{F}_{B}$ relative to $\mathcal{F}_{A}$, expressed in $\mathcal{F}_{C}$. The vector norm is the Euclidean norm denoted as $\|\boldsymbol{x}\|=\langle\boldsymbol{x}, \boldsymbol{x}\rangle^{\frac{1}{2}}$. The set of quaternions is defined as

$$
\mathbb{H}:=\left\{\left(q_{0}, \boldsymbol{q}_{v}\right): q_{0} \in \mathbb{R}, \boldsymbol{q}_{v} \in \mathbb{R}^{3}\right\}
$$

where $q_{0}$ is the scalar part and $\boldsymbol{q}_{v}$ is the vector part, while the set of unit quaternions is defined as $\mathbb{H}_{u}:=$ $\{\boldsymbol{q} \in \mathbb{H}:\|\boldsymbol{q}\|=1\}$. The set of vector quaternions is defined as $\mathbb{H}_{v}:=\left\{\boldsymbol{q} \in \mathbb{H}: q_{0}=0\right\}$.

The reference frames that are used in this work are defined as

Inertial frame This reference frame denoted as $\mathcal{F}_{i}$ has its origin at a fixed point in space and its axes are fixed.

Body frame This coordinate reference frame denoted as $\mathcal{F}_{b}$ is fixed at the rigid-body's center of mass and the axes are fixed to the rigid-body.

Desired frame This coordinate reference frame denoted as $\mathcal{F}_{d}$ represents the rigid-body's desired pose.

\section{B. Quaternions}

In this section a brief overview of unit quaternions are given, more complete formulations can be found in literature $c f$. [13], [14]. Commonly unit quaternions are constructed as

$$
\boldsymbol{q}=\left[\begin{array}{ll}
\cos \frac{\theta}{2} & \boldsymbol{u}_{\theta}^{\top} \sin \frac{\theta}{2}
\end{array}\right]^{\top}
$$

where $\theta \in \mathbb{R}$ is the angle of rotation and $\boldsymbol{u}_{\theta} \in \mathbb{R}^{3}$ is a unit vector pointing in the direction of axis of rotation. A vector $\boldsymbol{u} \in \mathbb{R}^{3}$ can obviously be extended to $\mathbb{H}_{v}$ and this fact is implicitly used in this paper. A vector $\boldsymbol{u}^{b} \in \mathbb{H}_{v}$ can be rotated from $\mathcal{F}_{b}$ to $\mathcal{F}_{n}$ by the use of the sandwich product

$$
\boldsymbol{u}^{n}=\boldsymbol{q}_{n b} \otimes \boldsymbol{u}^{b} \otimes \boldsymbol{q}_{n b}^{*}
$$

where $\boldsymbol{u}^{n} \in \mathbb{H}_{v}$ and $q_{n b} \in \mathbb{H}_{u}$ represents the orientation of frame $\mathcal{F}_{b}$ with respect to $\mathcal{F}_{n}$ and where $(\cdot) \otimes(\cdot)$ denotes the quaternion product and is defined as

$$
\boldsymbol{q}_{1} \otimes \boldsymbol{q}_{2}=\left[\begin{array}{c}
q_{1,0} q_{2,0}-\boldsymbol{q}_{1, v}^{T} \boldsymbol{q}_{2, v} \\
q_{1,0} \boldsymbol{q}_{2, v}+q_{2,0} \boldsymbol{q}_{1, v}+\boldsymbol{q}_{1, v} \times \boldsymbol{q}_{2, v}
\end{array}\right]
$$

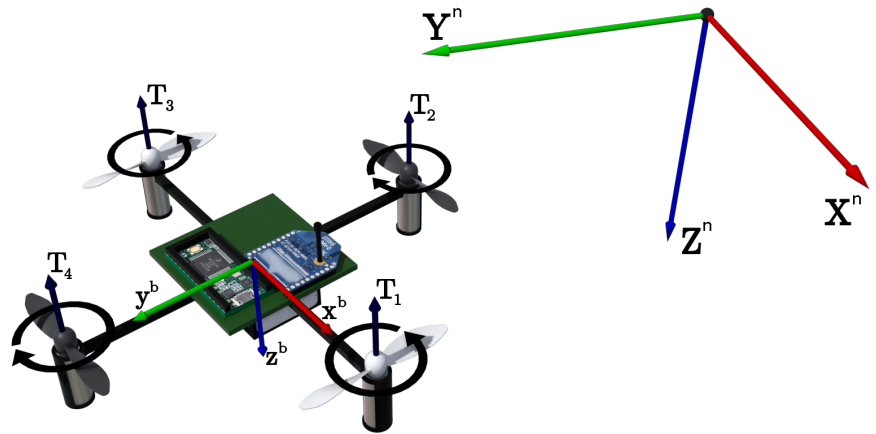

Fig. 1: Illustration of reference frames often used in quadrotor literature c.f. [9].

which is associative and distributive, but not commutative and $(\cdot)^{*}$ denotes the quaternion conjugate $\boldsymbol{q}^{*}=\left[\begin{array}{ll}q_{0} & -\boldsymbol{q}_{v}^{\top}\end{array}\right]^{\top}$. The norm of a quaternion can be defined as

$$
\|\boldsymbol{q}\|=\sqrt{\boldsymbol{q}^{\top} \boldsymbol{q}}
$$

and should always be equal to unity for a unit quaternion to ensure that lengths are preserved when using (2). Quaternions can be combined using the quaternion product to represent composite rotations as

$$
\boldsymbol{q}_{n d}=\boldsymbol{q}_{n b} \otimes \boldsymbol{q}_{b d}
$$

and difference in rotations can be defined as

$$
\boldsymbol{q}_{b d}=\boldsymbol{q}_{n b}^{*} \otimes \boldsymbol{q}_{n d} .
$$

The quaternion kinematics is defined as [13]

$$
\dot{\boldsymbol{q}}_{n b}=\frac{1}{2} \boldsymbol{q}_{n b} \otimes \boldsymbol{\omega}_{n b}^{b}
$$

where $\boldsymbol{\omega}_{n, b}^{b} \in \mathbb{R}^{3}$ is the angular velocity of $\mathcal{F}_{b}$ relative to $\mathcal{F}_{n}$, expressed in $\mathcal{F}_{b}$.

\section{Modeling}

\section{A. Quadrotor kinematics and dynamics}

In the quadrotor dynamics literature c.f. [15], [16], [9], the Newton-Euler equations of motion, with reference frames as shown in Figure 1, for a quadrotor is commonly defined as

$$
\begin{aligned}
\dot{\boldsymbol{p}}^{n} & =\boldsymbol{v}^{n} \\
\dot{\boldsymbol{q}}_{n b} & =\frac{1}{2} \boldsymbol{q}_{n b} \otimes \boldsymbol{\omega}_{n b}^{b} \\
\dot{\boldsymbol{v}}^{n} & =\frac{1}{m}\left(\boldsymbol{f}_{G}^{n}-\boldsymbol{q}_{n b} \otimes \boldsymbol{f}_{T}^{b} \otimes \boldsymbol{q}_{n b}^{*}\right) \\
\dot{\boldsymbol{\omega}}_{n, b}^{b} & =\left(\boldsymbol{J}^{b}\right)^{-1}\left(\boldsymbol{\tau}_{u}^{b}-\boldsymbol{\omega}_{n, b}^{b} \times\left(\boldsymbol{J}^{b} \boldsymbol{\omega}_{n, b}^{b}\right)\right)
\end{aligned}
$$

where $p^{n} \in \mathbb{R}^{3}$ is the inertial position of the quadrotor, $\boldsymbol{v}^{n} \in \mathbb{R}^{3}$ is the quadrotor's inertial velocity, $\boldsymbol{f}_{G}^{n} \in \mathbb{R}^{3}$ is the gravity vector, $\boldsymbol{q}_{n b} \in \mathbb{H}_{u}$ is the orientation of the quadrotor parameterized using quaternions, $\boldsymbol{\omega}_{n, b}^{b} \in \mathbb{R}^{3}$ is the angular velocity of the quadrotor, $\boldsymbol{J}^{b} \in \mathbb{R}^{3 \times 3}$ is the inertia matrix, $\boldsymbol{f}_{T}^{b}=\left[\begin{array}{lll}0 & 0 & T\end{array}\right]^{\top}$ is the quadrotor's thrust vector and $\tau^{b} \in \mathbb{R}^{3}$ is the applied torques on the quadrotor. The 
total thrust is defined as the sum of the thrusts $T_{i} \in \mathbb{R}$ as seen in Figure 1, that each rotor generates

$$
T=\sum_{i=1}^{4} T_{i}=\sum_{i=1}^{4} c_{T} \sigma_{i}^{2}
$$

which is related to the rotor's angular velocity $\sigma_{i} \in \mathbb{R}$ through the thrust coefficient $c_{T} \in \mathbb{R}$. From Figure 1 it is clear that the input torques acting on the quadrotor are also related to the thrust of each rotor by [15]

$$
\begin{aligned}
& \tau_{x}=d_{r} T_{2}-d_{r} T_{4}=d_{r} c_{T} \sigma_{2}^{2}-d_{r} c_{T} \sigma_{4}^{2} \\
& \tau_{y}=d_{r} T_{1}-d_{r} T_{3}=d_{r} c_{T} \sigma_{1}^{2}-d_{r} c_{T} \sigma_{3}^{2} \\
& \tau_{z}=c_{Q} \sigma_{1}^{2}+c_{Q} \sigma_{3}^{2}-c_{Q} \sigma_{2}^{2}-c_{Q} \sigma_{4}^{2}
\end{aligned}
$$

where $\sigma_{i}$ for $i=1,2,3,4$ is the angular velocity of the ith propeller, $c_{Q} \in \mathbb{R}$ is the torque coefficient and $d_{r} \in \mathbb{R}$ is the distance from the center of the quadrotor to the center of the propellers which is assumed to be equal for all the rotors. Since the equations are linear in $\sigma_{i}$ they can be represented by a linear mapping from angular velocities of each motor to applied thrust and torque as

$$
\left[\begin{array}{c}
T \\
\tau_{x} \\
\tau_{y} \\
\tau_{z}
\end{array}\right]=\left[\begin{array}{cccc}
c_{T} & c_{T} & c_{T} & c_{T} \\
0 & d_{r} c_{T} & 0 & -d_{r} c_{T} \\
d_{r} c_{T} & 0 & -d_{r} c_{T} & 0 \\
c_{Q} & -c_{Q} & c_{Q} & -c_{Q}
\end{array}\right]\left[\begin{array}{c}
\sigma_{1}^{2} \\
\sigma_{2}^{2} \\
\sigma_{3}^{2} \\
\sigma_{4}^{2}
\end{array}\right] .
$$

The mapping from rotor angular velocities to applied force and torque on quadrotor is of full-rank and therefore inverse mapping exists at all times since $c_{T}, c_{Q}$ and $d_{r}$ are constants.

\section{Control}

In this section a positional controller that assumes the translational dynamics are fully actuated is developed using the well known backstepping approach [17], [18], [3]. The resulting fully actuated control force vector is then saturated and injected into the attitude dynamics together with a dampening term that ensures boundedness on the angular velocities. Then the fully actuated control force vector is mapped to the total thrust $T$.

\section{A. Translational control}

Given a desired constant position defined by $\boldsymbol{p}_{d}^{n} \in \mathbb{R}^{3}$ the position error is defined as

$$
\tilde{\boldsymbol{p}}^{n}=\boldsymbol{p}_{d}^{n}-\boldsymbol{p}^{n}
$$

with the error kinematics

$$
\dot{\tilde{\boldsymbol{p}}}^{n}=\tilde{\boldsymbol{v}}^{n}=-\boldsymbol{v}^{n}
$$

and the following assumptions

Assumption 1: The mass $m$ of the quadrotor is assumed to be known.

Assumption 2: The attitude of the quadrotor $\boldsymbol{q}_{n b}$ is assumed to be known.
Assumption 3: The quadrotor is assumed to be fullyactuated i.e. the control force in (7) is replaced by a fully actuated control force $\boldsymbol{f}_{u}^{n}$.

Theorem 1. Consider a Quadrotor as shown in Figure 1 with its kinematics and dynamics described by (5)-(8) together with Assumption 1, Assumption 2 and Assumption 3. Given a desired constant position $\boldsymbol{p}_{d}^{n}$, define the error kinematics as in (15). If the input force $\boldsymbol{f}_{u}^{n}$ is given by

$$
\boldsymbol{f}_{u}^{n}=\boldsymbol{f}_{G}^{n}-\left(1+k_{p} k_{d}\right) \tilde{\boldsymbol{p}}^{n}+\left(m k_{p}+k_{d}\right) \boldsymbol{v}^{n} .
$$

then $\left(\tilde{\boldsymbol{p}}^{n}, \tilde{\boldsymbol{v}}^{n}\right) \rightarrow(\mathbf{0}, \mathbf{0})$ as $t \rightarrow \infty$ for any initial condition.

Proof: Consider the radially unbounded Lyapunov function candidate

$$
V=\frac{1}{2} \tilde{\boldsymbol{p}}^{n \top} \tilde{\boldsymbol{p}}^{n}+\frac{m}{2}\left(k_{p} \tilde{\boldsymbol{p}}^{n}+\tilde{\boldsymbol{v}}^{n}\right)^{\top}\left(k_{p} \tilde{\boldsymbol{p}}^{n}+\tilde{\boldsymbol{v}}^{n}\right)
$$

taking the derivative of (17) and inserting (15), (7) and (16) yields

$$
\dot{V}=-k_{p}\left\|\tilde{\boldsymbol{p}}^{n}\right\|^{2}-k_{d}\left\|k_{p} \tilde{\boldsymbol{p}}^{n}+\tilde{\boldsymbol{v}}^{n}\right\|^{2} \leq-V<0
$$

making the equilibrium of the closed loop system with the backstepping controller globally asymptotically stable.

\section{B. Attitude control}

The developed translational controller $\boldsymbol{f}_{u}^{n}$ in (16) requires that the translational system is fully actuated and is therefore not realizable for a quadrotor. To realize the control input the vector $\boldsymbol{f}_{u}^{n}$ is partitioned into a desired direction and desired thrust magnitude. An attitude controller is then designed to point the quadrotor's body z-axis along the axis of the direction of $\boldsymbol{f}_{u}^{b}$. This usually requires an attitude tracking controller that tracks a desired attitude designed from $\boldsymbol{f}_{u}^{b}$ and is therefore susceptible to complications due to the topological obstructions of the $\mathrm{SO}(3)$ manifold [8]. The proposed attitude controller circumvents this issue by injecting the control force $\boldsymbol{f}_{u}^{b}$ into the attitude system as a torque. This is achieved by first saturating the control force $f_{b}^{n}$ in (16) with for instance a saturation function defined as

$$
\tilde{\boldsymbol{f}}_{u}^{n}=\alpha\left(1-e^{-k_{f}\left\|\boldsymbol{f}_{u}^{n}\right\|}\right) \frac{\boldsymbol{f}_{u}^{n}}{\sqrt{\left\|\boldsymbol{f}_{u}^{n}\right\|^{2}+\Delta^{2}}}
$$

or

$$
\tilde{\boldsymbol{f}}_{u}^{n}=\left\{\begin{array}{cl}
\boldsymbol{f}_{u}^{n} & \text { if } k_{f}\left\|\boldsymbol{f}_{u}^{n}\right\|<\alpha \\
\alpha \frac{\boldsymbol{f}_{u}^{n}}{\sqrt{\left\|\boldsymbol{f}_{u}^{n}\right\|^{2}+\Delta^{2}}} & \text { if } k_{f}\left\|\boldsymbol{f}_{u}^{n}\right\| \geq \alpha
\end{array}\right.
$$

where $\Delta$ is a positive constant that avoids division by zero if $\left\|\boldsymbol{f}_{u}^{n}\right\|=0, \alpha \in \mathbb{R}$ represents the maximum torque the control vector can inject into the attitude system and $k_{f} \in \mathbb{R}$ is a tuning parameter that affects how quickly $\boldsymbol{f}_{u}^{b}$ gets saturated. In both cases of (19) and (20), $\tilde{\boldsymbol{f}}_{T}^{n}$ is bounded from above by $\alpha>0$.

Theorem 2. Consider a Quadrotor as shown in Figure 1 with its kinematics and dynamics described by (5)-(8) together with Assumption 1, Assumption 2. Suppose a force vector $\tilde{\boldsymbol{f}}_{u}^{n}$ is 
calculated from (16) and saturated using (19) or (20) and let the input torque be defined as

$$
\boldsymbol{\tau}_{u}^{b}=-\boldsymbol{e}_{3} \times\left(\boldsymbol{q}_{n b}^{*} \otimes \tilde{\boldsymbol{f}}_{u}^{n} \otimes \boldsymbol{q}_{n b}\right)-k_{\omega} \boldsymbol{\omega}_{n b}^{b} .
$$

Then the torque $\boldsymbol{\tau}_{u}^{b}$ is bounded by $\alpha$, and $\boldsymbol{\omega}_{n b}^{b}$ is bounded by $\frac{\alpha}{k_{\omega}}$.

Proof: Let a Lyapunov function candidate $V$ be defined

as

$$
V=\frac{1}{2}\left(\boldsymbol{\omega}_{n b}^{b}\right)^{\top} \boldsymbol{J} \boldsymbol{\omega}_{n b}^{b}
$$

which is positive definite and radially unbounded. The derivative can be written as

$$
\dot{V}=\left(\boldsymbol{\omega}_{n b}^{b}\right)^{\top} \boldsymbol{J} \dot{\boldsymbol{\omega}}_{n b}^{b}
$$

and inserting (8) and (21) yields

$$
\begin{aligned}
\dot{V}= & \left(\boldsymbol{\omega}_{n b}^{b}\right)^{\top}\left(-\boldsymbol{e}_{3} \times\left(\boldsymbol{q}_{n b}^{*} \otimes \tilde{\boldsymbol{f}}_{u}^{n} \otimes \boldsymbol{q}_{n b}\right)\right. \\
& \left.-k_{\omega} \boldsymbol{\omega}_{n b}^{b}-\boldsymbol{\omega}_{n b}^{b} \times \boldsymbol{J} \boldsymbol{\omega}_{n b}^{b}\right) .
\end{aligned}
$$

Using the fact that $\left(\boldsymbol{\omega}_{n b}^{b}\right)^{\top} \boldsymbol{\omega}_{n b}^{b} \times \boldsymbol{J} \boldsymbol{\omega}_{n b}^{b}=\mathbf{0}$ in (24) yields

$$
\begin{aligned}
\dot{V}= & -\frac{1}{2} k_{\omega}\left\|\boldsymbol{\omega}_{n b}^{b}\right\|^{2}-\left\|\boldsymbol{\omega}_{n b}^{b}\right\|\left\|\tilde{\boldsymbol{f}}_{u}^{n}\right\| \sin \theta_{1} \cos \theta_{2} \\
& -\frac{1}{2} k_{\omega}\left\|\boldsymbol{\omega}_{n b}^{b}\right\|^{2}
\end{aligned}
$$

where $\theta_{1}$ is the angle between $\boldsymbol{e}_{3}$ and $\tilde{\boldsymbol{f}}_{u}^{b}$, and $\theta_{2}$ is the angle between $\boldsymbol{e}_{3} \times\left(\boldsymbol{q}_{n b}^{*} \otimes \boldsymbol{f}_{u}^{n} \otimes \boldsymbol{q}_{n b}\right)$ and $\boldsymbol{\omega}_{n b}^{b}$. Since the inner angle between two vectors is $\theta_{1} \in[0, \pi]$ such that $\sin \theta_{1} \in[0,1]$ then

$$
\dot{V} \leq-\frac{1}{2} k_{\omega}\left\|\boldsymbol{\omega}_{n b}^{b}\right\|^{2}-\left\|\boldsymbol{\omega}_{n b}^{b}\right\|\left\|\tilde{\boldsymbol{f}}_{u}^{n}\right\| \cos \theta_{2}-\frac{1}{2} k_{\omega}\left\|\boldsymbol{\omega}_{n b}^{b}\right\|^{2} .
$$

Completing the squares in (26) yields

$$
\begin{aligned}
\dot{V} & \leq-\frac{1}{2} k_{\omega}\left\|\boldsymbol{\omega}_{n b}^{b}\right\|^{2}-\left(\sqrt{\frac{k_{\omega}}{2}}\left\|\boldsymbol{\omega}_{n b}^{b}\right\|+\frac{1}{2} \sqrt{\frac{2}{k_{\omega}}}\left\|\tilde{\boldsymbol{f}}_{u}^{n}\right\| \cos \theta_{2}\right)^{2} \\
& +\frac{1}{2 k_{\omega}}\left\|\tilde{\boldsymbol{f}}_{u}^{n}\right\|^{2}\left(\cos \theta_{2}\right)^{2} \\
& \leq-\frac{1}{2} k_{\omega}\left\|\boldsymbol{\omega}_{n b}^{b}\right\|^{2}+\frac{1}{2 k_{\omega}}\left\|\tilde{\boldsymbol{f}}_{u}^{n}\right\|^{2} .
\end{aligned}
$$

From (19) and (20) it clear that $\left\|\tilde{\boldsymbol{f}}_{u}^{b}\right\|$ is bounded by $\alpha$ such that

$$
\dot{V} \leq-\frac{1}{2} k_{\omega}\left\|\boldsymbol{\omega}_{n b}^{b}\right\|^{2}+\frac{\alpha^{2}}{2 k_{\omega}}
$$

which is negative definite when

$$
\frac{\alpha}{k_{\omega}}<\left\|\omega_{n b}^{b}\right\|
$$

which implies that $\boldsymbol{\omega}_{n b}^{b}$ is bounded. Inserting the bounds on $\boldsymbol{\omega}_{n b}^{b}$ and (19) shows that $\boldsymbol{\tau}_{u}^{b}$ is bounded by $2 \alpha$. It can be seen from (21) the attitude controller does not require knowledge about the inertia matrix, only of the total mass of the quadrotor from (16) is needed. The effect the term $\boldsymbol{e}_{3} \times \tilde{f}_{u}^{b}$ has on the attitude system is that it causes a tilting motion towards the direction of $\boldsymbol{f}_{u}^{b}$. This causes $\left\|\boldsymbol{f}_{u}^{b}\right\|$ to decrease since the quadrotor is thrusting in this direction such that the bound on the body angular velocities will also decrease. Additionally both $\theta_{1}$ and $\theta_{2}$ will also decrease such that $\left\|\boldsymbol{\omega}_{n b}^{b}\right\|^{2}$ will dominate $\left\|\boldsymbol{\omega}_{n b}^{b}\right\|\left\|\tilde{\boldsymbol{f}}_{u}^{n}\right\| \sin \theta_{1} \cos \theta_{2}$ and the angular velocities will decrease. The angular velocity will only go to zero when $\sin \theta_{1}$ goes to zero. When $\sin \theta_{1}$ is non-zero the attitude controller will try and tilt the quadrotor towards $\boldsymbol{f}_{u}^{b}$. This leads to the quadrotor converging to the desired position.

\section{Thrust control}

The magnitude of $\boldsymbol{f}_{u}^{b}$ needs to be mapped to the actual thrust vector of the quadrotor in (7). This is done by relating $\boldsymbol{f}_{u}^{b}$ to the total thrust $T$ of the quadrotor. There are several possible ways to define the thrust $T$ from $\boldsymbol{f}_{u}^{n}$, for instance if the thrust $T$ is defined as

$$
T=\boldsymbol{e}_{3}^{\top} \boldsymbol{q}_{n b}^{*} \otimes \boldsymbol{f}_{u}^{n} \otimes \boldsymbol{q}_{n b}
$$

then the translation control becomes a height controller that gives suitable transients for position control. However the controller can yield negative thrust values which is not realizable unless the quadrotor can vary the pitch of the propellers, it is therefore necessary to saturate the thrust to zero when it reaches negative values. Defining the thrust $T$ as

$$
T=\left\|\boldsymbol{f}_{u}^{n}\right\|
$$

together with high values of $k_{p}$ and $k_{d}$ yields a more aggressive controller that can give better results for trajectory tracking. The reasoning for this is that the controller will always yield positive thrust even if the quadrotor is pointing away from the desired position. For instance if quadrotor is in hover and the desired position is below the quadrotor, it will start thrusting away from the desired position until the attitude controller flips the quadrotor upside down towards the desired position.

\section{Simulation}

In this section the quadrotor dynamics in (7) and (8) are simulated with the position controller (16) and attitude controller (21). Two cases are presented where in case one position control is explored and in case two a circle tracking example is demonstrated. In both cases the physical data of the quadrotor is found in Table I

\section{A. Case 1: Position control}

The gain parameters were chosen as $k_{p}=2, k_{v}=1, k_{\omega}=$ $3, \alpha=5, \Delta=0.1, k_{f}=0.5$. The desired position is defined as

$$
\boldsymbol{p}_{d}^{n}=\left[\begin{array}{lll}
5 & 5 & -10
\end{array}\right]^{\top}
$$

TABLE I: Inertia parameters for a quadrotor [19]

\begin{tabular}{|l|l|l|}
\hline Parameter & Value & Unit \\
\hline Quadrotor mass, $m$ & 4.34 & $\mathrm{~kg}$ \\
X-axis inertia, $J_{x x}$ & 0.082 & $\mathrm{~kg} \cdot \mathrm{m}^{2}$ \\
Y-axis inertia, $J_{y y}$ & 0.0845 & $\mathrm{~kg} \cdot \mathrm{m}^{2}$ \\
Z-axis inertia, $J_{z z}$ & 0.1377 & $\mathrm{~kg} \cdot \mathrm{m}^{2}$ \\
\hline
\end{tabular}




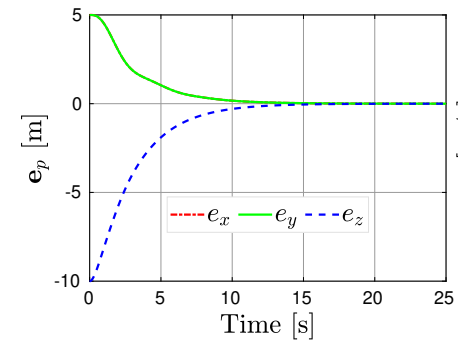

(a) Position error.

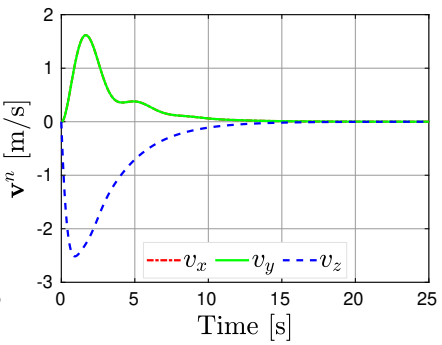

(b) Inertial velocities.
Fig. 2: Translational motion of case 1.

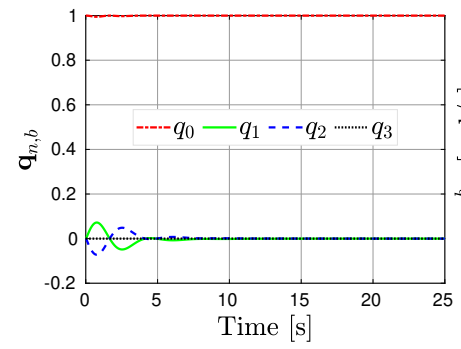

(a) Quadrotor attitude.

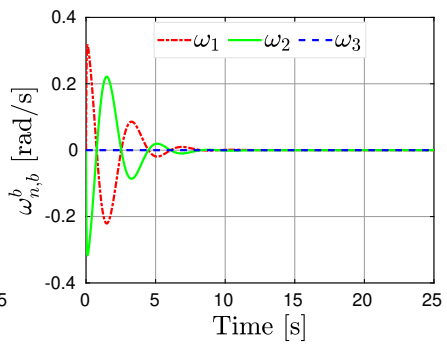

(b) Quadrotor angular velocities.
Fig. 3: Rotational motion of case 1.

with initial conditions set to

$$
\begin{aligned}
\boldsymbol{q}_{n, b}(0) & =\left[\begin{array}{llll}
1 & 0 & 0 & 0
\end{array}\right]^{\top}, \quad \boldsymbol{\omega}_{n, b}^{b}(0)=\left[\begin{array}{lll}
0 & 0 & 0
\end{array}\right]^{\top}[\mathrm{rad} / \mathrm{s}] \\
\boldsymbol{p}^{n}(0) & =\left[\begin{array}{lll}
0 & 0 & 0
\end{array}\right]^{\top}[\mathrm{m}], \quad \boldsymbol{v}^{n}(0)=\left[\begin{array}{lll}
0 & 0 & 0
\end{array}\right]^{\top}[\mathrm{m} / \mathrm{s}] \\
\sigma_{i}(0) & =0[\mathrm{rad} / \mathrm{s}], \quad i=1,2,3,4 .
\end{aligned}
$$

As can be seen from Figure 2a the position error converges to zero. The angular velocities in Figure $3 \mathrm{~b}$ are within the bound of $\frac{5}{3}$ and the torques in Figure 4 are within $\alpha$. There are some oscillations in the start on the angular velocity which is related to the $-\boldsymbol{v}$ in (16) such that if the quadrotor accelerates too much the control vector will start to point away from the desired position and cause the quadrotor to tilt away from the desired position to slow down. At the start in Figure 4 the torques are initially at $\tau_{u}^{b}=\left[\begin{array}{lll}1 & -1 & 0\end{array}\right]^{\top}$ since it is dependent on the desired position and initial conditions of the translational system.

\section{B. Case 2: Circle tracking}

In this case the physical limitations of the proposed controller is explored by injecting large disturbances on states used in the controllers. The quadrotor is also made to follow a trajectory by augmenting the backstepping controller in (16) to be

$$
\boldsymbol{f}_{u}^{n}=\boldsymbol{f}_{G}^{n}-m \ddot{\boldsymbol{p}}_{d}^{n}-\left(1+k_{p} k_{d}\right) \tilde{\boldsymbol{p}}^{n}-\left(m k_{p}+k_{d}\right) \tilde{\boldsymbol{v}}^{n}
$$

where the errors are now defined as

$$
\begin{gathered}
\tilde{\boldsymbol{p}}^{n}=\boldsymbol{p}_{d}^{n}-\boldsymbol{p}^{n} \\
\tilde{\boldsymbol{v}}^{n}=\dot{\boldsymbol{p}}_{d}^{n}-\boldsymbol{v}^{n} .
\end{gathered}
$$

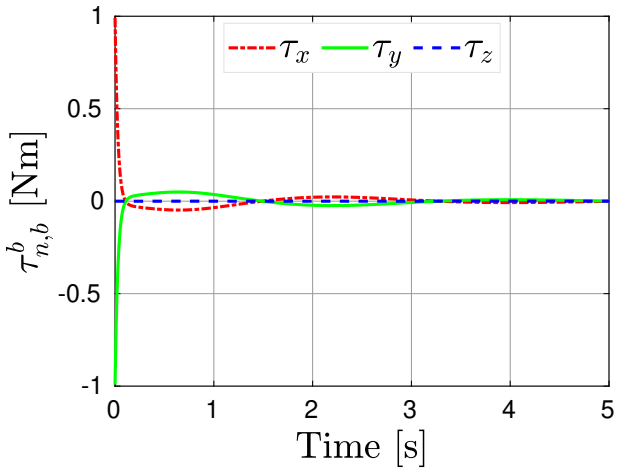

Fig. 4: Quadrotor control torque of case 1

and the desired trajectory is assumed to be at least twice differentiable and bounded i.e. $\boldsymbol{p}_{d}^{n}, \dot{\boldsymbol{p}}_{d}^{n}, \ddot{\boldsymbol{p}}_{d}^{n} \in \mathcal{L}_{\infty}$. A trajectory that satisfies these requirements is given as

$$
\begin{aligned}
& \boldsymbol{p}_{d}^{n}=\left[\begin{array}{lll}
c_{1} \sin c_{2} t & c_{1} \cos c_{2} t & c_{3}-c_{4} \sin c_{5} t
\end{array}\right]^{\top}[\mathrm{m}] \\
& \dot{\boldsymbol{p}}_{d}^{n}=\left[\begin{array}{lll}
c_{1} c_{2} \cos c_{2} t & -c_{1} c_{2} \sin c_{2} t & -c_{4} c_{5} \cos c_{5} t
\end{array}\right]^{\top}[\mathrm{m} / \mathrm{s}] \\
& \ddot{\boldsymbol{p}}_{d}^{n}=\left[\begin{array}{lll}
-c_{1} c_{2}^{2} \sin c_{2} t & -c_{1} c_{2}^{2} \cos c_{2} t & c_{4} c_{5}^{2} \sin c_{5} t
\end{array}\right]^{\top}\left[\mathrm{m} / \mathrm{s}^{2}\right]
\end{aligned}
$$

where $c_{1}=10 \mathrm{~m}, c_{2}=0.15 \mathrm{rad} / \mathrm{s}, c_{3}=-10 \mathrm{~m}, c_{4}=0.0 \mathrm{~m}$ and $c_{5}=0.0 \mathrm{rad} / \mathrm{s}$. The initial conditions were set as

$$
\begin{aligned}
& \boldsymbol{q}_{n, b}=\left[\begin{array}{llll}
1 & 0 & 0 & 0
\end{array}\right]^{\top}, \quad \boldsymbol{\omega}_{n, b}^{b}=\left[\begin{array}{lll}
0 & 0 & 0
\end{array}\right]^{\top}[\mathrm{rad} / \mathrm{s}] \\
& \boldsymbol{p}^{n}=\left[\begin{array}{lll}
0 & 0 & 0
\end{array}\right]^{\top}[\mathrm{m}], \quad \boldsymbol{v}^{n}=\left[\begin{array}{lll}
0 & 0 & 0
\end{array}\right]^{\top}[\mathrm{m} / \mathrm{s}] \\
& \sigma_{i}=0[\mathrm{rad} / \mathrm{s}], \quad i=1,2,3,4 .
\end{aligned}
$$

In addition a yaw tracking controller was added to the attitude system where the kinematics of a desired pointing trajectory is defined as $\dot{\boldsymbol{b}}^{n}=\bar{\omega}_{z} \boldsymbol{e}_{3} \times \boldsymbol{b}^{n}$ with yaw orientation error defined as $\tilde{\boldsymbol{b}}=\boldsymbol{q}_{n b} \otimes \boldsymbol{e}_{1} \otimes \boldsymbol{q}_{n b}^{*} \times \boldsymbol{b}^{n}$ where both $\boldsymbol{q}_{n b} \otimes \boldsymbol{e}_{1} \otimes \boldsymbol{q}_{n b}^{*}$ and $\boldsymbol{b}^{n}$ have been projected to the $x y$-plane. Then the yaw controller $\boldsymbol{\tau}_{\text {yaw }}^{b}=k_{e} \tilde{b}+k_{\omega}\left(\bar{\omega}_{z}-\boldsymbol{\omega}_{n b}^{b \top} \boldsymbol{e}_{3}\right) \boldsymbol{e}_{3}$ makes the equilibrium of the error system asymptotically stable. The desired angular velocity $\bar{\omega}_{z}$ was set to $2 \mathrm{rad} / \mathrm{sec}$ and bandlimited white noise with power spectral density of $5 \cdot 10^{-6}$ was added on all state variables in the control laws. As can be seen from Figure 6a the position error seem to go to zero. However, the position error converge to around $2 \cdot 10^{-1} \mathrm{~m}$ which implies that the body $z$-axis never fully aligns itself with the force control vector in (34). This is expected because of the added noise and that the attitude controller does not ensure trajectory tracking. However, the performance of the tracking can be improved by increasing $k_{p}$ at the expense of increased oscillations on $\boldsymbol{\omega}_{n b}^{b}$ at the start of the simulation.

\section{CONCLUSIONS}

In this paper a reaction-based control strategy was developed for a simplified model of a quadrotor. The proposed controller has several advantages such as not requiring the knowledge of the inertia matrix and the controller avoids issues related to the topology of $\mathrm{SO}(3)$ by not tracking a desired attitude. Simulations were performed which shows that the 


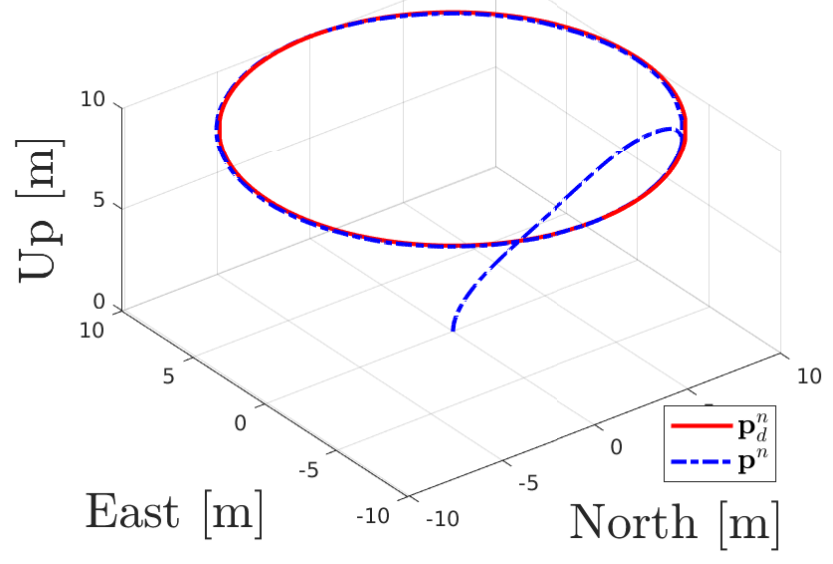

Fig. 5: Trajectory plot of case 2.

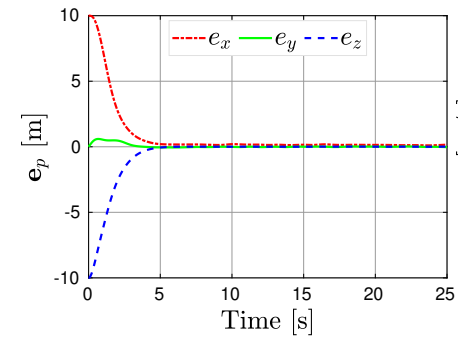

(a) Position error.

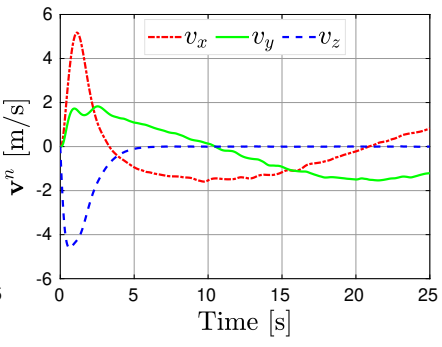

(b) Inertial velocities.
Fig. 6: Translational motion of case 2.

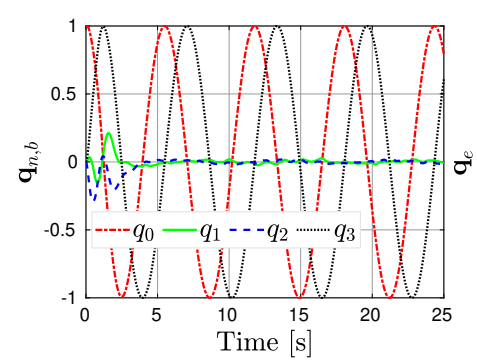

(a) Angular velocities.

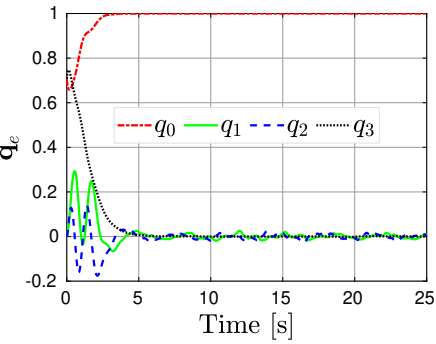

(b) Applied torques.
Fig. 7: Rotational motion of case 2.

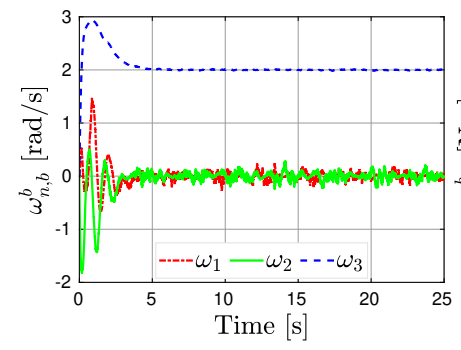

(a) Angular velocities.

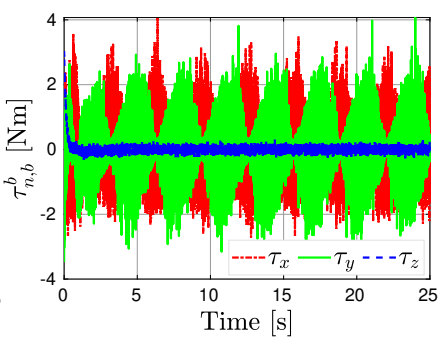

(b) Applied torques.
Fig. 8: Angular velocity and applied torques of case 2 . quadrotor converges to desired positions and it also shows that the proposed method can do trajectory tracking in cases with large disturbances in the quadrotor states. Future work will include testing the controller in experiments, adding an adaptive position controller that can estimate the quadrotor mass, and improving the proposed controllers disturbance rejection and tracking capabilities.

\section{ACKNOWLEDGMENT}

This research has been funded by the Norwegian Research Council and is part of the Sikrere logistikk fra fartøy med ubemannet logistikkhelikopter project 282317.

\section{REFERENCES}

[1] K. Valavanis and G. J. Vachtsevanos, Eds., Handbook of Unmanned Aerial Vehicles. Springer Netherlands, 2015

[2] B. Siciliano and O. Khatib, Eds., Springer Handbook of Robotics. Springer International Publishing, 2016.

[3] T. P. Nascimento and M. Saska, "Position and attitude control of multirotor aerial vehicles: A survey," Annual Reviews in Control, vol. 48, pp. $129-146,2019$

[4] C. S. Subudhi and D. Ezhilarasi, "Modeling and trajectory tracking with cascaded pd controller for quadrotor," Procedia Computer Science, vol. 133, pp. 952 - 959, 2018, international Conference on Robotics and Smart Manufacturing (RoSMa2018).

[5] Q. Ye, "Event-driven pid control of autonomous quadrotor helicopters," Journal of Algorithms \& Computational Technology, vol. 12, no. 2, pp. 159-164, 2018.

[6] G. Cai, J. Dias, and L. Seneviratne, "A survey of small-scale unmanned aerial vehicles: Recent advances and future development trends," $U n$ manned Systems, vol. 02, no. 02, pp. 175-199, 2014.

[7] A. Roza and M. Maggiore, "A class of position controllers for underactuated vtol vehicles," IEEE Transactions on Automatic Control, vol. 59, no. 9, pp. 2580-2585, 2014.

[8] S. P. Bhat and D. S. Bernstein, "A topological obstruction to continuous global stabilization of rotational motion and the unwinding phenomenon," Systems \& Control Letters, vol. 39, no. 1, pp. 63 - 70, 2000.

[9] M. D. Hua, T. Hamel, P. Morin, and C. Samson, "Introduction to feedback control of underactuated VTOL vehicles: A review of basic control design ideas and principles," IEEE Control Systems, vol. 33, no. 1, pp. 61-75, Feb 2013.

[10] R. R. Warier, A. K. Sanyal, S. Sukumar, and S. P. Viswanathan, "Feedback tracking control schemes for a class of underactuated vehicles in se(3)," in In Proc. of American Control Conference, 2017, pp. 899904.

[11] A. Sanchez, V. Parra-Vega, C. Tang, F. Oliva-Palomo, and C. IzaguirreEspinosa, "Continuous reactive-based position-attitude control of quadrotors," in In Proc. of American Control Conference, 2012, pp. $4643-4648$

[12] C. Izaguirre-Espinosa, A. Muñoz-Vázquez, A. Sánchez-Orta, V. ParraVega, and G. Sanahuja, "Fractional attitude-reactive control for robust quadrotor position stabilization without resolving underactuation," Control Engineering Practice, vol. 53, pp. 47 - 56, 2016.

[13] O. Egeland and J. T. Gravdahl, Modeling and Simulation for Automatic Control. Marine Cybernetics, 2002.

[14] E. B. Dam, M. Koch, and M. Lillholm, "Quaternion, interpolation and animation," University of Copenhagen, Tech. Rep., 1998.

[15] R. Mahony, V. Kumar, and P. Corke, "Multirotor aerial vehicles: Modeling, estimation and control of quadrotor," IEEE Robotics Automation Magazine, vol. 19, no. 3, pp. 20 - 32, 2012.

[16] S. Bouabdallah and R. Siegwart, "Full control of a quadrotor," in International Conference on Intelligent Robots and Systems, 2007, pp. $153-158$.

[17] M. Krstić, I. Kanelllakopoulos, and P. Kokotović, Nonlinear and Adaptive Control Design. John Wiley \& Sons, 1995.

[18] A. P. Aguiar and J. P. Hespanha, "Position tracking of underactuated vehicles," in Proceedings of the 2003 American Control Conference, 2003., vol. 3, June 2003, pp. 1988-1993. 
[19] P. Pounds, R. Mahony, and P. Corke, "Modelling and control of a large quadrotor robot," Control Engineering Practice, vol. 18, no. 7, pp. 691 - 699, 2010, special Issue on Aerial Robotics. 